Paediatr. Paedolog. 2021 · 56:126-129 https://doi.org/10.1007/s00608-021-00895-5 Angenommen: 21. April 2021 Online publiziert: 8 . Juni 2021

(๑) Der/die Autor(en) 2021

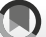

\section{Einleitung}

Es ist ja wirklich ganz einfach: Psyche bedeutet in Altgriechisch „Hauch, Atem, Seele“ und Soma „Körper, Leib“. Die Betrachtung von Phänomenen, die beide Lebenssysteme gleichzeitig betreffen, ist erforderlich. Beim diagnostischen und therapeutischen Prozess soll man sich gleichwertig den messbaren Parametern und den erahnten Signalen zuwenden. Heute werden dafür auch gern die Begriffe holistisch oder ganzheitlich verwandt. Man meint damit die Berücksichtigung von mehreren, auch transkulturellen Sichtweisen als nur die, die die westliche akademische Medizin lehrt. Je erfahrener und älter Ärzte werden, desto offener und großzügiger wird bei vielen diese Haltung, was sich in einem wachsenden Interesse für die Psychosomatik zeigt.

Interaktion in technischer Weise beschrieben: Die Zwischenhandlung, die sich auf die Arzt-Patient-Beziehung und im Fall der Pädiatrie auf Faktoren bezieht, die die Begegnung des Helfers mit dem Mutter-Kind- dem Vater-Kind-Paar oder der Eltern-Kind-Triade meint. Darunter kann man alle beziehungswirksamen Phänomene subsummieren sowie den Wirkfaktor Arzt selbst.

Wie präsentieren wir dieses aktuelle Thema interaktiv: in einem Dialog zwischen den Autoren. Es sollte dieser Dialog bestenfalls einen inneren Dialog im Leser auslösen (• Abb. 1).

Bei einem Besuch in Iximche, einer Maya Stadt in der guatemaltekischen Hochebene, kamen wir ungeplant zu dem einmal jährlich stattfindenden Event des „hombre des mais“. Dies ist eine Dank- und Gebetszeremonie für den Mais, den Lebensspender. Die Zeremonie ist ein originäres Kulturevent der

\author{
Marguerite Dunitz-Scheer ${ }^{1}$ Peter Scheer ${ }^{2}$ \\ 'Firma Notube.com, Graz, Österreich \\ ${ }^{2} \mathrm{Graz}$, Österreich
}

\title{
Psychosomatik und Interaktion
}

indigenen Bevölkerung und beinhaltet eine medizinische Theorie, die auf der Farbsymbolik des Mais basiert. Mais kommt in der Natur in Weiß, Gelb, Rot und Schwarz vor. Weiß steht für Zähne und Knochen, Gelb für Haut, Rot fürs Blut und Herz, Schwarz für Haare und Augen. Wir sahen eine Stunde lang zu, wie die Bevölkerung in Kostümen in den vier Farben, Blumen- und Opfergaben darbrachte. Sie feierten das Fest mit Tänzen, Gebeten und Gesängen. Zum Schluss wurden in einem Feuer die Maiskolben verbrannt. Wir hatten währenddessen Zeit, uns Gedanken zu den unterschiedlichen Körper- und Gesundheitsmodellen $\mathrm{zu}$ machen. Unsere Reiseleiterin erklärte, dass die Farbtheorie auch heute noch eine wichtige Rolle in der Bevölkerung spielt. Obschon ihre Erklärungen für uns nicht schlüssig waren, weil uns „der Glaube“ fehlt, haben uns die Feierlichkeit und Ernsthaftigkeit der Mais-Zeremonie nachhaltig beeindruckt.

\section{BEGINN DES DIALOGS (kursiv: P. J. Scheer)}

Kommunikation findet immer statt (Paul Watzlawik). Jede Interaktion in einer kinderärztlichen Praxis ist schon Kommunikation. Die Art des Empfangs, der Zustand und Besuch des Wartezimmers (es darf nicht zu leer und nicht zu voll sein) spielen eine Rolle, lange bevor der Arzt oder die Ärztin in die Kommunikation eintritt.

Weiterhin: Es gibt keinen Körper ohne „das Ich“. Darum gibt es auch kein "Ist das organisch, oder ist das psychisch?", sondern man hat immer mit dem ganzen Menschen zu tun. Alle Formulierungen, die einen der angeblich zwei Teile (Körper oder Kopf) des Menschen ansprechen, stö- ren mich. Wie kann es sein, dass Läufer von: „Es kommt alles vom Kopf!" sprechen, wenn sie ihre Motivation meinen? Wie kann es sein, dass Kinderärzte von einer organischen Krankheit sprechen, wo doch das Kind als Ganzes betroffen ist. Egal, ob seine Schmerzen aus der Angst entstehen oder Schmerzen Angst machen?

Natürlich ist die Idee der Maya-Kultur für uns bizarr, wie viele religiösen Schöpfungs- und Heilungsideen. Sie erscheinen uns absurd, obwohl wissenschaftlich gesehen zwischen der Mais-Theorie und Hahnemanns Theorie der "Ähnlichkeit“ kein Unterschied besteht. Selbst in den sogenannten entwickelten Ländern mit guter wissenschaftlicher Kultur wird an die Homöopathie geglaubt, sie viel verordnet und angewandt. Ich kann und will nicht über den Maismenschen urteilen, lieber urteile ich mit (Ingomar D. Mutz) über den Unsinn der Mondphasen und deren angeblichem Einfluss aufs Haare-

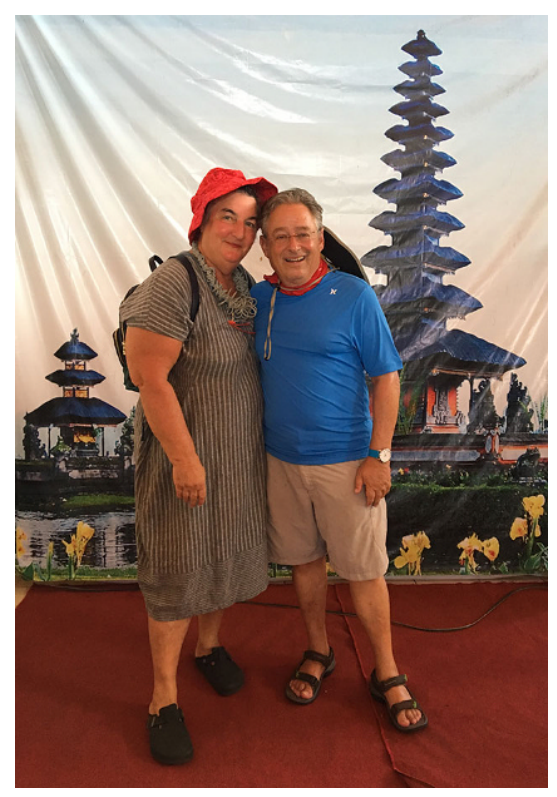

Abb. $1 \Delta$ Das Ehe- und Autorenpaar bei der Ankunft in Mexiko (C P. Scheer) 
schneiden, als dass ich mich über andere Kulturen lustig mache.

Wenn wir es nett finden, eine andere Kultur zu beobachten, so mag es auch ersprießlich sein, neue Kommunikationsund Interaktionsformen auszuprobieren. Vielleicht einmal den Lernenden oder den Zuhörenden oder den Lobenden oder den Unwissenden - das was wir nicht gut können - anwenden. Da stellt sich uns der größte Feind entgegen: die eigene Persönlichkeit und Gewohnheit. Ein Arzt, der immer als Wissendender aufgetreten ist, wird schwer eine andere Rolle annehmen, weil Wissender sein Erfolgsrezept ist. Er begründet das mit seinem vollen Wartezimmer oder mit den dankbaren Patienten. Er übersieht dabei alle anderen Handlungs- und Verhaltensoptionen. Vielleicht ist eine Reise nach Guatemala doch ein guter Anfang einen kleinen Schritt aus seiner Routine zu machen und Neues zu probieren.

ANTWORT: Nun, jetzt bin ich (MDS) wieder dran. Mein Mann behauptet, ich „hätte es“ - ganz im Gegensatz zu ihm mehr mit dem Faktischen: mit Zeiten, Orientierung, Daten. Genauso wie bei meinem Umgang in der Pädiatrie: was, wie lange, wie viel, warum und wann. Er beschäftigt sich - so wie ich das sehe - lieber mit konstruktivistischer Philosophie und diffusen Eindrücken und Ahnungen. Damit meint man, soweit ich es verstehe, dass wir nur wahrnehmen, was wir wahrnehmen können/wollen und dass „nur“ diese subjektiven Erfahrungen unsere Auffassung der Realität formen. Modern definiert, die sensorische Integration formt die kognitive Wahrnehmung.

EIN BEISPIEL: Ich sehe jetzt eine normale Praxissituation vor mir: Ein Elternteil, meist die Mutter, kommt mit ihrem fiebernden Kind in die Praxis. Krankheit kommt immer ungelegen; unsere Mutter hat zum Beispiel noch ein zweites, älteres Kind, das vom Kindergarten abgeholt werden muss. Sie selbst fühlt sich auch krank, vielleicht hat sie denselben Infekt, den ihr größeres Kind vor einer Woche nach Hause gebracht hat. Der Empfang verläuft nicht ideal, denn sie fragt: „Wie lange wird's denn dauern, bis ich dran bin?" Die mehrfach beschäftigte Dame am Empfang sagt nun zum Beispiel: „Sehen's eh!"Der Dialog, in dem die Mama einbringen wollte, dass sie in einer Stunde ihren Großen vom Kindergarten abzuholen hat, ist entglitten. Ein: „Ich kann mich aber jetzt wirklich nicht einreihen, ich muss ja - sagen wir Georg zu ihm - in einer Stunde abholen.", fand nicht statt. Denn die vorher phantasierte Antwort, wie immer sie auch ausfallen mag, ist oft nicht hilfreich. Die Mutter setzt sich neben die anderen Mütter mit deren kranken Kindern ins Wartezimmer. Ihr Kind beginnt sofort zu jammern. Sie zieht es aus und fürchtet, dass ihr Kleines hier noch kränker werden könnte. Als sie aufgerufen wird, hat sie es schon sehr eilig. Die Ärztin, die an dem Tag schon viele Infekte gesehen hat, liest die Anamnese am Computer: „Ah, “ sagt sie, „ein Infekt.“ Selbst ein unverfängliches: „Was kann ich für Sie tun?" das ich oft empfohlen habe, wäre besser, aber beim vorhandenen Zeitbudget vielleicht unangemessen. Vielleicht ist meine Szene noch $z$ nett, vielleicht findet der Arzt das Kind bereits ausgezogen in einer der Untersuchungsräume vor und greift das Kind gleich an. Untersucht - Kind schreit -, findet nicht viel, schaut sich das Blutbild an, das im Vorfeld gemacht wurde, verordnet etwas oder auch nicht und sagt, dass die Mama bei jeder Verschlechterung des Zustands des Kindes nochmals vorbeikommen soll.

Ist die Begegnung gelungen? Hat man genug gehört, gesehen? Weiß man wirklich, weshalb die Mutter in die Praxis gekommen ist? Oder sind wir den kurzen Weg gegangen, der von unbesprochenen Annahmen ausgeht?

KOMMENTAR: Ich finde das Beispiel gut und typisch. Die meisten Kinder, die in die Praxis gebracht werden, sind glücklicherweise nicht ernstlich krank. Unsere Aufgabe und Verantwortung ist es darum, die „richtig“ Kranken rauszupicken, $\mathrm{zu}$ behandeln und bei Bedarf weiterzuschicken. Alle anderen muss man so korrekt, sensibel und liebevoll wie möglich möglichst rasch „loswerden“, aber auch hierfür gibt's klare Anweisungen.

Georg war jedenfalls kein schwer krankes Kind in einem bedrohlichen Zustand, sondern die typische und häufige Situation, in der sich die Mutter die Mühe machte, in der Praxis vorbeizukommen, weil sie beunruhigt war, obwohl sie Stress hatte. Sie wollte nur
Paediatr. Paedolog. $2021 \cdot$ 56:126-129 https://doi.org/10.1007/s00608-021-00895-5 (c) Der/die Autor(en) 2021

\section{Dunitz-Scheer ·P. Scheer \\ Psychosomatik und Interaktion}

\section{Zusammenfassung}

In diesem kleinen Beitrag versuchen die Autoren, beide aus dem Bereich der pädiatrischen Psychosomatik kommend, ihre Erlebnisse bezüglich der Medizin, die sie bei einer Weltreise 2019 hatten, darzustellen. Dabei geht es um den Maismenschen ebenso wie um die Kraft der Göttin des Vulkans.

Die Anwendung auf unseren Erlebniskreis ist schwierig und wird hier ansatzweise versucht.

\section{Schlüsselwörter}

Psychosomatik - Der Maismensch · Pele · Die Vulkangöttin Hawaiis · Göttin des Mais

\section{Psychosomatics and Interaction}

\section{Abstract}

In this short article, the authors, both from the field of pediatric psychosomatic medicine, attempt to describe the medicalrelated experiences that they had during a round-the-world trip in 2019. It concerns the maize men as well as the power of the goddess of volcanoes. Applying this to our sphere of experience is difficult and is attempted here in a rudimentary manner.

\section{Keywords}

Psychosomatics - Hombre de Mais - Pele . Godness of vulcano in Hawaii - Goddess of volcanoes

wissen, ob man das Fieber großzügig oder sparsam senken sollte oder ob und ab wann man an die Gabe eines Antibiotikums denken sollte oder ob sie womöglich sogar dem Kind geschadet hätte, weil sie mit ihm in die volle Praxis gekommen war? Wir wissen es nicht, denn die „eigentliche Frage“ (Parzivals Frage: Was wirret Dich? ${ }^{1}$ ) wurde nicht gestellt.

1 Der Held der von Wolfram aufgezeichneten Geschichte Parzival kommt nach einigen Mühen als der designierte, neue Herrscher zur Gralsburg. Die Bedingung, Gralskönig 
Es ist hilfreich und wichtig, dass man für diese täglichen Normalsituationen die Interaktionsqualität, trotz Trubel, reflektiert und schaut, ob die Mutter, einfühlsam und respektvoll begrüßt und verabschiedet wurde und ob sie bekommen hat, was sie wollte, ob man sozusagen eine ausreichend gute Kundenzufriedenheit erreicht hat. Dazu bedarf es nicht nur der Selbstreflexion, sondern auch einer unablässigen Teamschulung, gerade auch „schwierige“ oder „lästige“ Patienten mit Zuvorkommenheit zu behandeln, denn alles andere (Unkonzentriertheit, Nicht-Ernst-Nehmen, Ärger, Vorwürfe, Beschimpfungen) erzeugt zusätzlichen Stress.

Das gute alte Rezept, sich „ganz einfach" in die Rolle der Mutter einzufühlen oder sich vorzustellen, die junge Mutter wäre die eigene Tochter, die mit ihrem kranken Kind einen Kollegen besucht, hilft sehr. Die Fähigkeit und Gabe, jeden Patienten erfreut und positiv zu begrüßen, sodass die Familie wiederkommen wird, ist viel wert.

Insofern ist jede Begegnung in der pädiatrischen Praxis zumindest auch eine psychosomatische! Selbst wenn das Kind einen ganz klar „somatischen“ Vorstellungsgrund wie einen Ausschlag oder erhöhte Körpertemperatur hat, ist die Qualität der Untersuchungssituation entscheidend, um die richtige Diagnose zu finden und gute Behandlung zu machen. „Ende gut, alles gut" ist darum erst erreicht, wenn das Motiv zur Vorstellung erkannt wird. Erst wenn die Gleichung „Anamnese + Befund führen zu Diagnose und Behandlung “ gelöst ist, stimmt's. Damit das geschieht kann, müssen andere Faktoren genauso berücksichtigt werden wie die Hautfarbe oder die Körpertemperatur oder das Blutbild.

zu werden, ist Mitgefühl mit dem an einer Geschlechtskrankheit leidenden König zu haben. Parzival sollte Amfortas fragen: „Was wirret Dich?" Aus falscher Rücksicht und wegen falscher Ritterlichkeit unterlässt er das. Nun muss er auf weite Reisen gehen, die fast sein ganzes Leben dauern, um Mitgefühl zu lernen. Im Jahr 1980 habe ich (PS) deshalb Parzival zum „idealtypischen“ psychosomatischen Patienten in dem Buch: H. Zimprich: Kinderpsychosomatik (Stuttgart, Thieme, 1980) gemacht, weil er seinem Gefühl keinen Ausdruck verleihen kann.
Gelebte Psychosomatik ist eine Haltung, die sich in Begegnungen ausdrückt. Obwohl wir Ärztinnen und keine Priesterinnen sind, sollten wir Patienten ihre Wahrnehmungen schildern lassen. Ist das geschehen, kann man die Eindrücke sammeln und eine Diagnose komponieren. Alles Weitere ergibt sich dann „wie von selbst".

DAZU NOCH: So schön es ist, dass sich Marguerite anhand der Eindrücke, die sie von außen bekommt, orientiert, so schwer ist es, das in die Alltagspraxis eines Kinderarztes zu übersetzen. Die zentrale Aussage ist einfach: wahrnehmen und wachsam sein. Insofern verstehe ich Marguerite. Sie möchte Sie sensibilisieren für all' das, was um Sie herum passiert. Verstehen, wahrnehmen, interagieren - aber heute ist alles scheinbar nur genetisch determiniert. Plötzlich sind wir wieder in der Zeit der Monarchie, wo das „Blut" alle Chancen determiniert und der Handlungsspielraum des Einzelnen kaum gegeben war. Daher finde ich, dass es fein wäre, sich nicht nur damit zu beschäftigen, was gerade "in" (früher sagte man: „en vogue") ist. So schön die DNA ist, so toll die neuen Techniken: Die Begegnung mit dem Patienten und seiner Umgebung bleibt bedeutend. Gelingt sie, ist Freude der Lohn.

\section{Korrespondenzadresse}

Univ.-Prof. Dr. Marguerite Dunitz-Scheer Firma Notube.com

Kerschhoferweg 14, 8010 Graz, Österreich marguerite.dunitz-scheer@medunigraz.at

Funding. Open access funding provided by Medical University of Graz.

\section{Einhaltung ethischer Richtlinien}

Interessenkonflikt. M. Dunitz-Scheer und P. Scheer geben an, dass kein Interessenkonflikt besteht.

Für diesen Beitrag wurden von den Autoren keine Studien an Menschen oder Tieren durchgeführt. Für die aufgeführten Studien gelten die jeweils dort angegebenen ethischen Richtlinien.

\section{Anhang}

Da es praktisch kein gut lesbares Lehrbuch der psychosomatischen Medizin gibt, möchte ich Ihnen die Lektüre des
Klassikers Irren ist menschlich von Klaus Dorner und Ursula Plog ans Herz legen, der bereits 2017 in seiner 24. Auflage erschienen ist, sowie des Buchs Kinderpsychosomatik von Hans Zimprich.

Weitere Bücher, die sich aus meiner Sicht auch beeindruckend gut mit dem Thema auseinandersetzen, sind:

- Irvin Yalom: Die Liebe und ihr Henker und andere Geschichten aus der Psychotherapie. Übersetzt von Hans J. Heckler. Knaus, München 1990, ISBN 3-8135-6979-9

- Zeruya Shalev: Mann und Frau. Aus dem Hebräischen von Mirjam Pressler. Berlin-Verlag, Berlin 2001, ISBN 3-8270-0397-0.

- Batya Gur: Denn am Sabbat sollst du ruhen. Goldmann, München, 1992. ISBN 3-442-46008-5.

- John Bowlby: Bindung - Eine Analyse der Mutter-Kind-Beziehung. Kindler Verlag, 1982, ISBN 3-463-00618-9. (Originaltitel: Attachment and Loss. 1969/1983)

Open Access. Dieser Artikel wird unter der Creative Commons Namensnennung 4.0 International Lizenz veröffentlicht, welche die Nutzung, Vervielfältigung, Bearbeitung, Verbreitung und Wiedergabe in jeglichem Medium und Format erlaubt, sofern Sie den/die ursprünglichen Autor(en) und die Quelle ordnungsgemäß nennen, einen Link zur Creative Commons Lizenz beifügen und angeben, ob Änderungen vorgenommen wurden.

Die in diesem Artikel enthaltenen Bilder und sonstiges Drittmaterial unterliegen ebenfalls der genannten Creative Commons Lizenz, sofern sich aus der Abbildungslegende nichts anderes ergibt. Sofern das betreffende Material nicht unter der genannten Creative Commons Lizenz steht und die betreffende Handlung nicht nach gesetzlichen Vorschriften erlaubt ist, ist für die oben aufgeführten Weiterverwendungen des Materials die Einwilligung des jeweiligen Rechteinhabers einzuholen.

Weitere Details zur Lizenz entnehmen Sie bitte der Lizenzinformation auf http://creativecommons.org/ licenses/by/4.0/deed.de.

Hinweis des Verlags. Der Verlag bleibt in Hinblick auf geografische Zuordnungen und Gebietsbezeichnungen in veröffentlichten Karten und Institutsadressen neutral. 
Hier steht eine Anzeige.

\section{曾 Springer}

